\title{
Mechanical behaviour of graphdiyne film: experimental and molecular dynamics simulation
}

\author{
Kailu Xiao ${ }^{1,2}$, Xianqian $\mathrm{Wu}^{1,2, *}$, Qiuyun $\mathrm{Yin}^{3}$, Chenguang Huang ${ }^{1,2}$ \\ ${ }^{1}$ Key Laboratory of Mechanics in Fluid Solid Coupling Systems, Institute of Mechanics, Chinese Academy of Sciences, Beijing 100190, \\ China. \\ ${ }^{2}$ School of Engineering Science, University of Chinese Academy of Sciences, Beijing 100049, China. \\ ${ }^{3}$ School of Engineerin, Sun Yat-Sen University, Guangzhou 510275, China.
}

\begin{abstract}
AFM experiments and molecular dynamics simulation of rectangular graphdiyne films are performed in this paper. The force-deflection curves are obtained, and the elastic modulus is calculated as 218.5 GPa and 482.615 GPa, respectively. The simulated maximum stress and pre-tension of graphdiyne film are $33.088 \mathrm{GPa}$ and $0.551 \mathrm{GPa}$, respectively. It is observed that the graphdiyne film fractured in the central point once the indentation depth over the critical depth. Also, the obviously elastic behaviour has found during the loading-unloading-reloading process. The deformation mechanisms and fractured behaviour of the graphdiyne film are discussed in detail during the loading process. Moreover, the effects of various factors including loading speed and indenter radii of the graphdiyne film by the MD simulation are discussed.
\end{abstract}

\section{Introduction}

The two-dimensional materials have attracted great attention once graphene is discovered. Their superlative properties and potential applications such as electronic, chemical, thermal, mechanical stability and nanostructures have been a subject of extensive research recently [1-2]. Carbon's three hybridization states $\left(\mathrm{sp}^{3}\right.$, $\mathrm{sp}^{2}, \mathrm{sp}$ ), can be combinations to produce numerous carbon allotropes; such as diamond $\left(\mathrm{sp}^{3}\right)$, graphite $\left(\mathrm{sp}^{2}\right)$, fullerene $\left(\mathrm{sp}^{2}\right)$, carbon nanotubes $\left(\mathrm{sp}^{2}\right)$ and graphene $\left(\mathrm{sp}^{2}\right)$. Most of these materials have remarkable mechanical strength [3] and electronic properties [4]. Here, we pay attention to graphdiyne [5], one of the carbon allotropes with the satisfactory candidates of novel graphene-like materials in future. Graphdiyne, which is sp2-sp-hybridized carbon atoms and can be considered as one-third of the carbon-carbon bonds of graphene's crystal structure are replaced by one acetylene unit.

The structure of graphdiyne has diacetylene $(-\mathrm{C} \equiv \mathrm{C}-\mathrm{C} \equiv \mathrm{C}-$ ) linkages between benzene rings, which makes the more flexible than graphene because of graphdiyne's smaller density of atoms, indicating that the ductility of graphdiyne is better than graphene. Lee et al. [6] measured the intrinsic strength and in-plane stiffness of graphene are $130 \pm 10 \mathrm{GPa}$ and $1 \pm 0.1 \mathrm{TPa}$ at room temperature. Kang et al. [7] using the density functional theory (DFT) method calculated that the inplane stiffness of graphyne is $166 \mathrm{~N} / \mathrm{m}$, which is twice smaller than that of graphene. That means, graphyne is much flexible than graphene. Zhao et al. [8] using molecular simulation had found the ultimate tensile strength of graphene decrease with increasing temperature. Fang at al. [9] calculated the maximum Cauchy stress is $110 \mathrm{GPa}$ in the armchair direction and $121 \mathrm{GPa}$ in the zigzag direction of graphene using ab inito calculations. Cranford et al. [10] predicted the inplane stiffness of the monolayer graphdiyne is $170.4 \mathrm{~N} / \mathrm{m}$ and the strength is $45 \mathrm{GPa}$ in zigzag direction and $29 \mathrm{GPa}$ in armchair direction using molecular dynamics (MD). The study by Ahangari at al. [11] indicated that the in-plane stiffness and Young's modulus of graphdiyne decrease from $164.4 \mathrm{~N} / \mathrm{m}$ and $513.8 \mathrm{GPa}$ at room temperature to $128.9 \mathrm{~N} / \mathrm{m}$ and $402.8 \mathrm{GPa}$ at $1500 \mathrm{~K}$, respectively, using ab initio MD simulation. It's shown that the ductility of graphyne and graphdiyne is better than graphene but the in-plane strength of graphene is superior to. However, the deformation mechanism of graphyne is not clear up to now.

In this paper, the mechanical behaviour of graphdiyne sheet transferred to copper network with rectangular wells is investigated by AFM and MD simulation. The van der Waals interaction between copper network and graphdiyne can make the graphdiyne binding strongly with copper network. It shows excellent mechanical performance such as high strength, more flexible and stretchable when compared to graphene. Also, a detailed fracture behaviour of graphdiyne is investigated with MD simulation, which clear show that the fracture of graphdiyne's bonds leads to great ductility of graphdiyne.

\section{METHODS}

* Corresponding author: wuxianqian@imech.ac.cn 


\subsection{Materials}

The graphdiyne is fabricated with the same method as $\mathrm{Li}$ et al. [5]. The monomer of hexaethynylbenzene was synthesized in good yield (62\%) by adding tetrabutylammonium fluoride (TBAF) to the THF solution of hexakis[(trimethylsilyl)ethynyl]benzene for 10 minutes at $8{ }^{\circ} \mathrm{C}$. The graphdiyne grow on the surface of copper foil in the pyridine by a cross-coupling reaction of the monomer structure of hexaethynylbenzene for $72 \mathrm{~h}$ at $60{ }^{\circ} \mathrm{C}$ under nitrogen atmosphere. In the procedure of forming graphdiyne, the copper foil is not only the catalyst for the cross-coupling reaction but also the substrate for growing graphdiyne film, as shown in Fig. 1. After successfully synthesized the graphdiyne membrane, the film is transferred to a copper network with rectangular wells for preparing atomic force microscope (AFM) tests. The van der Waals interaction between copper network and graphdiyne can make the graphdiyne binding strongly with copper network. The length and width of the wells are both $6.1 \mu \mathrm{m}$. In AFM experiments, a diamond AFM tip was chosen. Electron micrographs of the tip are shown in Figure 3, the length of the indenter is $6.4 \pm 1$ $\mu \mathrm{m}$ and the radius of curvature is $20 \mathrm{~nm}$. The thermal noise method was used to calibrate the force constant of tip to be $25.47 \mathrm{~N} / \mathrm{m}$ by measuring the sensitivity of optical leverage system. The GDY membranes were scanned in the noncontact mode and a slightly withdraw were executed.

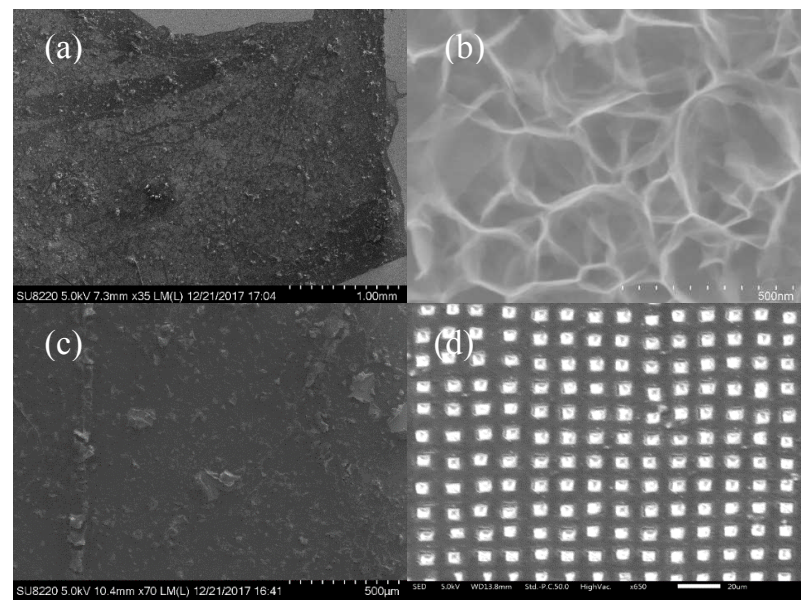

Fig. 1. SEM images of GDY film (a) GDY films grown on the surface of copper foil; (b) the microstructure of GDY film; (c) cracked film on the edge of copper foil; (d) GDY film after transferred to the copper network with rectangular well.

\subsection{MD model}

We perform molecular dynamics (MD) simulations through large scale atom/molecular massively parallel simulation (LAMMPS) package [12] to simulate the nanoindentation of a suspended sheet of GDY membranes. The number of samples carbon atoms is 81468. There are three constituent bonds: aromatic (e.g. $\mathrm{sp}^{2}$ ), single and triple (e.g. $\mathrm{sp}^{1}$ ), we set the bond length of $1.44 \AA$ for aromatic bonds, $1.40 \AA$ for single bonds and $1.24 \AA$ for triple bonds [13]. Fig. 2 shows the original model of our nanoindentation tests and the upper sphere configuration is the indenter. The indenter constructed by diamond, which is considered as a rigid object so that the indenter's configuration of atoms had no changes during MD simulations. The radii of indenter were set as $20 \AA$ so we can consider the contact area between indenter and membranes were point.

The boundary atoms are set clamped. The carboncarbon interaction of the GDY membrane and indenter atoms was described by adaptive intermolecular reactive empirical bond order (AIREBO) potential [14]. The interaction between indenter and GDY membrane were described by Lennard-Jones (LJ) potential. All the MD simulations were performing by canonical ensemble (i.e. NVT) and the temperature were controlled by NoseHoover and the room temperature of $300 \mathrm{~K}$ were set to be equilibrium temperature. Before simulate the process of nanoindention, the energy minimization and relaxation should be carried out to ensure the system stay in the equilibrium state with lowest energy.

Fig. 2. Atomic configuration of the original model.

\section{RESULTS AND DISCUSSION}

\subsection{Mechanical model}

According to the model of clamped circular elastic films under point load, the loading force $F$ and deflection $\delta$ can be approximated with a polynomial function[15]. We considered two conditions getting the expression fitted to the force-displacement relationship:

$$
F=F_{\sigma^{2 D}}(\delta)+F_{E^{2 D}}(\delta)
$$

The first term represents the membrane with a large initial pre-tension, and the second term is valid for stresses larger than the initial axial pre-tension. Based on our experiments condition, the first term can be written as

$$
F_{\sigma^{2 D}}(\delta)=\sigma_{0}^{2 D}(\pi a h) \beta^{1 / 2}\left(\frac{r}{a}\right)^{3 / 4}\left(\frac{\delta}{a}\right)
$$

where $\sigma_{0}^{2 D}$ is the pre-tension of membrane, $\beta$ is the aspect ratio of the sample, and $a$ represents the equivalent radius of the rectangular GDY membrane,

The second term can be written as

$$
F_{E^{2 D}}(\delta)=E^{2 D}\left(q^{3} a h\right) \beta^{1 / 2}\left(\frac{r}{a}\right)^{1 / 4}\left(\frac{\delta}{a}\right)^{3}
$$

where $E^{2 D}$ is Young's modulus of two-dimensional materials, and $q$ is an dimensionless constant, and $v$ is Poisson's ratio, $v=0.4$.

According to Bhatia et al. [16], the maximum stress for a rectangular elastic film can be expressed as

$$
\sigma_{m}^{2 D}=\left(\frac{F E^{2 D}}{8 \pi R}\right)^{1 / 2}
$$


where $\sigma_{m}^{2 D}$ is the maximum stress of the membrane under indenter, $\mathrm{R}$ is the radii of indenter tip and $F$ is the breaking force under indentation.

\subsection{The results of AFM experiments}

Different thickness has been tests of the GDY film, so the dispersion of our data also proved the thickness has significantly effects to the elastic modulus. The measured relationship of load-deflection can be seen in Fig. 3. Because the thickness from $10 \mathrm{~nm}$ to $30 \mathrm{~nm}$ exists in our tests, the averaged elastic modulus is $218.5 \mathrm{GPa}$, which is almost one half of the MD results. This is due to the flaws in the synthetized film and the measured modulus is the defects materials. When the thickness increased, the elastic modulus also increased.

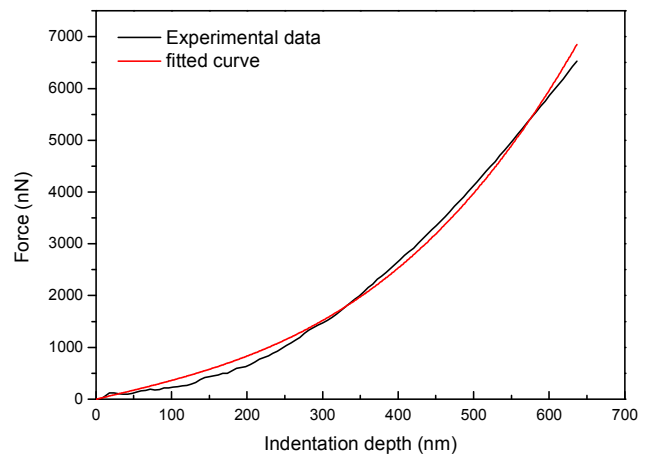

Fig. 3. Load and indentation depth curves for the experiment and fitted by Eq.(4).

\subsection{The results of MD simulation}

The indenter was placed over the center of the GDY membranes and moved perpendicular to the surface of the original GDY sheets with a constant velocity. The atoms on the edge of the GDY film kept in a static state because the edge atoms were fixed. After loading enough time and distance, some lattices of the GDY membranes were ultimately fractured and it shows the failure mode of the GDY membrane.

\subsubsection{Elastic modulus analysis}

The simulated force-deflection curve is given in Fig. 4. The case of indenter radii as $2 \mathrm{~nm}$ and loading velocity as $0.2 \AA / \mathrm{ps}$ was considered to illustrate this behaviour. The fitted curve is according to the nonlinear least squares methods based on formula (1). The maximum value of the indentation depth is $42.08 \AA$ corresponding to the $40.36 \mathrm{nN}$, which is the critical value of the GDY film broken.

The simulated elastic modulus, maximum stress and pre-tension of GDY are $482.615 \mathrm{GPa}, 33.088 \mathrm{GPa}$ and $0.551 \mathrm{GPa}$, respectively. The elastic modulus in our experiments is in good agreement with those published works [17], and the maximum stress is harmonious with the published work [10]. When the indentation depth is less than $4.22 \AA$, the slope of the curve is larger than the other range.

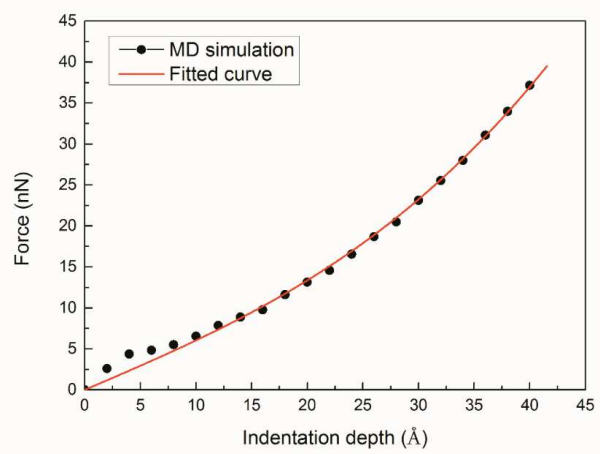

Fig. 4. Load and indentation depth curves for the MD simulation results.

\subsubsection{The fracture behaviour of GDY under indentation}

The loading- deflection curves can be obtained from the data of indenter load and indentation depth as shown in Fig. 5. It can see that the load suddenly dropped once the indentation depth over $42.08 \AA$ corresponding to the load $40.36 \mathrm{nN}$ when the loading velocity is $0.2 \AA / \mathrm{ps}$. It can be understood by the different number of bonds in graphene and GDY. Each $\mathrm{C}$ atom is three-fold coordinated of graphene, whereas in GDY, the average coordination number of $\mathrm{C}$ atom is 2.5 . This could be leading to the relatively smaller in-plane stiffness of GDY.

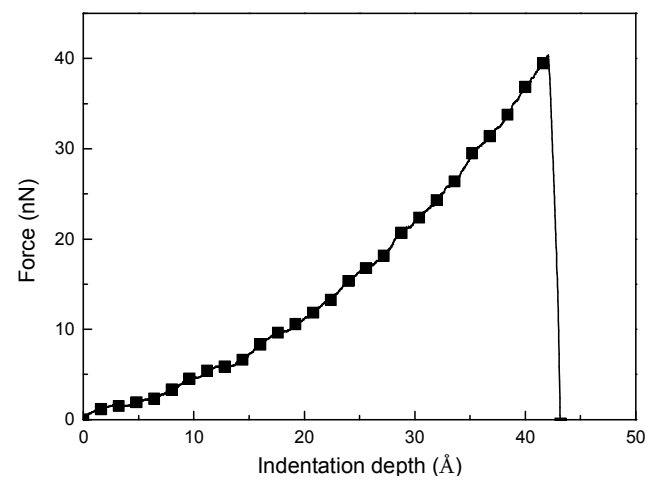

Fig. 5. The force-deflection curve of loading process.

The fracture behaviour happens when the load exceeded the ultimate force of GDY. Fig. 6 shows some detailed on the lattice fracture of the GDY film. The attractive pair potential exists in the indenter and film when the indenter close to the surface of GDY film, so that the distance small than $3.4 \AA$, the GDY film will be convex. The GDY film has a "snap-through" when the indenter contact with GDY film. With the increase of indentation depth, the force between indenter and GDY sheet changed from attractive interaction to repulsive interaction. Consequently, the force on the local atom which under indenter is non-uniform, the $\mathrm{C}$-C bonds getting larger and the GDY film still keep initial structure, as shown in Fig. 6(b). With the indentation depth increasing, the covalent bonds length getting change of the atoms surrounded by indenter, and the acetylenic bond broken firstly when the indentation depth over critical depth, the GDY film was expanded largely, as shown in Fig. 6(c). That is due to the energy 
of triple bond large than double bond and single bond, and the first fracture bond of triple bond is $\pi$ bond. Then the $\mathrm{C}=\mathrm{C}$ bonds broken subsequently, the fracture bond of double bond is also $\pi$ bond, after that, some $\mathrm{C}-\mathrm{C}$ bonds broken and that's due to the $\sigma$ bond is more stable than $\pi$ bond. When the GDY film is broken, the force between indenter and film suddenly dropped to 0 and the bondbroken is irreversible. When the indenter fully penetrate the GDY film, some broken atoms separated from the GDY film and it can't be recovery so that an irregular crack has occurred, as shown in Fig. 6(d). The broken $\mathrm{C}-\mathrm{C}$ bonds and $\mathrm{C} \equiv \mathrm{C}$ bond will re-combine to deform the circle structure, as shown in Fig. 6(e), the black circle part. Also, the quadrangular structure has appearance, as shown in Fig. 6(f), the blue circle part. The broken of $\mathrm{C}=\mathrm{C}$ bond is partial to combine with the near atoms and form different shape as shown in Fig. $6(\mathrm{~g})$, the circle part., the trilateral structure has shown in the red circle part. Be differ from the fracture of graphene [18], the GDY film deform a lathy crack through bond breaking and reforming as shown in Fig. 6(h). In near further the effects of indenter radii and loading speed on the mechanics properties of GDY film will be performed.
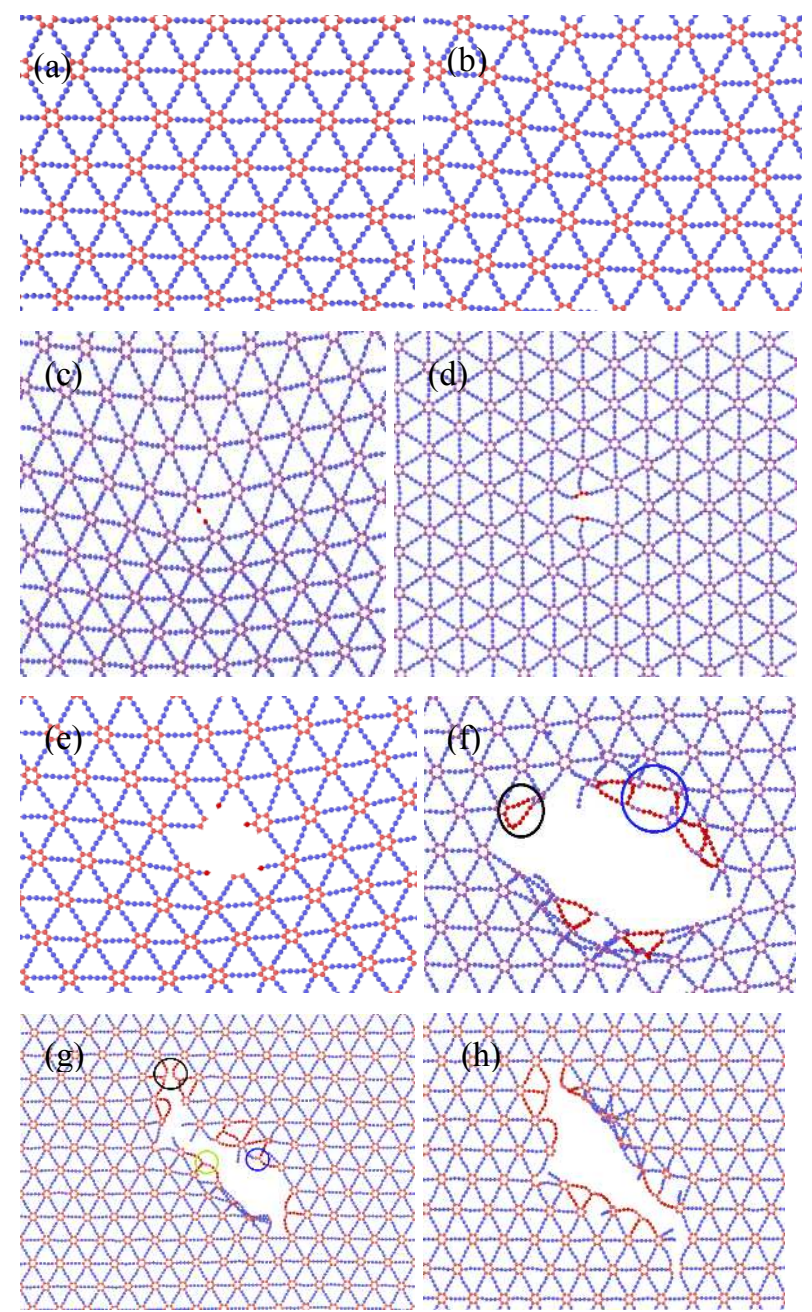

Fig. 6. The evolution of GDY film lattice fracture (a) the initial structure; (b) the prolonged lattice structure; (c) the first broken of acetylenic bond; (d) the second broken of the $\mathrm{C}=\mathrm{C}$ bonds; (e) The broken of the GDY film; (f) the re-combined structure of the $\mathrm{C}-\mathrm{C}$ bonds and $\mathrm{C} \equiv \mathrm{C}$ bonds as marked in the figure; $(\mathrm{g})$ the fracture manners of the $\mathrm{C}=\mathrm{C}$ bonds as marked in the figure; (h) the final broken behaviour of GDY film.

\section{CONCLUSION}

The mechanical behaviour of GDY film is studied by AFM tests and MD simulations in this paper. The microstructure of synthesized GDY film is obtained by scanning electron microscope (SEM) and then its elastic modulus is measured to be $218.5 \mathrm{GPa}$ by AFM experiments. An atomic model of GDY is constructed and the nanoindentation process is carried out. The maximum value of the indentation depth is $42.08 \AA$ corresponding to the $40.36 \mathrm{nN}$ in the case of indenter radii as $2 \mathrm{~nm}$ and loading velocity as $0.2 \AA / \mathrm{ps}$, the elastic modulus, maximum stress and pre-tension of GDY are 482.615 GPa, 33.088 GPa and 0.551 GPa, respectively. Once the indentation depth over the critical depth, the broken of the GDY film occur, and the failure behaviour of the GDY is analysed according to its micro-structures during continuous loading.

\section{References}

1 E. Mccann, V.I. Fal'Ko, Physical Review Letters, 96 086805 (2006)

2 K.S. Novoselov, E. Mccann, S.V. Morozov, V.I. Falko, M.I. Katsnelson, U. Zeitler, D. Jiang, F. Schedin, A.K. Geim, Nature Physics, 2 177-180 (2006)

3 A.H. Esbati, S. Irani, Archives of Civil \& Mechanical Engineering, 18 532-545 (2018)

4 M.S. Ribeiro, A.L. Pascoini, W.G. Knupp, I. Camps, Applied Surface Science, 426 (2017)

5 G. Li, Y. Li, H. Liu, Y. Guo, Y. Li, D. Zhu, Chem Commun (Camb), 46 3256-3258 (2010)

6 C. Lee, X. Wei, J.W. Kysar, J. Hone, Science, 321 385-388 (2008)

7 J. Kang, J. Li, F. Wu, S.S. Li, J.B. Xia, Journal of Physical Chemistry C, 115 20466-20470 (2011)

8 H. Zhao, N.R. Aluru, Journal of Applied Physics, 108 064321-064321-064325 (2010)

9 S.H. Chen, L. Liu, T.C. Wang, International Journal of Solids and Structures, 44 4492-4504 (2007)

10 S.W. Cranford, D.B. Brommer, M.J. Buehler, Nanoscale, 4 7797-7809 (2012)

11 M.G. Ahangari, Physica E: Low-dimensional Systems and Nanostructures, 66 140-147 (2015)

12 S. Plimpton, Fast parallel algorithms for short-range molecular dynamics (Academic Press Professional, Inc., 1995)

13 H. Bai, Y. Zhu, W. Qiao, Y. Huang, RSC Advances, 1768 (2011)

14 S.J. Stuart, A.B. Tutein, J.A. Harrison, The Journal of Chemical Physics, 112 6472-6486 (2000)

15 U. Komaragiri, M.R. Begley, J.G. Simmonds, Journal of Applied Mechanics, 72203 (2005)

16 N.M. Bhatia, W. Nachbar, AIAA Journal, 6 10501057 (1968)

17 J.I. Klingemann, Carbon, 49 4111-4121 (2011) 
18 W. Wang, S. Li, J. Min, C. Yi, Y. Zhan, M. Li, Nanoscale Research Letters, 9 (2014) 
